\section{Léon Van Hove}

Léon Van Hove, a prominent theoretical physicist and a former Research Director General of CERN (1976-1980), died on 2 September only 18 months after his formal retirement from the organization where he had worked since 1961.

Léon Van Hove received his Ph.D. in 1946 from the Université Libre de Bruxelles. After working in Brussels and at the Advanced Studies Institute in Princeton for a total of three years, he became one of the youngest professors in Europe when he accepted in 1954 the chair of theoretical physics at the University of Utrecht.

$\mathrm{He}$ is regarded as a principal architect of the postwar revival of research in physics in continental Europe. His teaching of quantum physics benefited a generation of young scientists who were desperately tying to learn quickly and in depth new approaches in theoretical physics.

He came to CERN to lead the Theory Division where his unique mastery of the whole research programme of the organization brought him quickly to wide ranging responsabilities. He was the Research Director General at a time when crucial decisions had to be made such as transforming the newly built SPS into a proton-antiproton collider, constructing for that purpose the first intense source of antimatter in the world and steering the LEP project towards approval. These developments lie at the

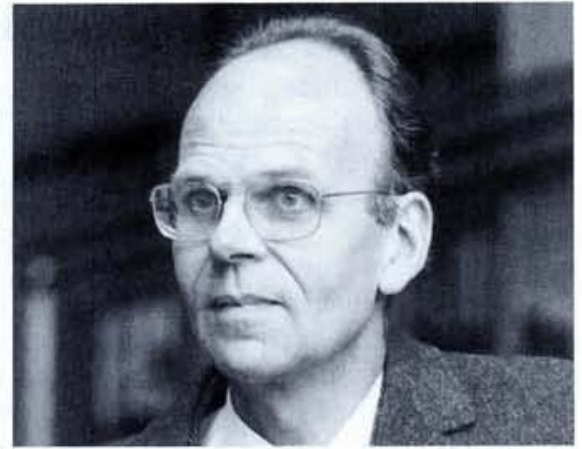

origin of the great successes in the eighties which have given CERN first place worldwide in particle physics.

Léon Van Hove's main contributions to physics span statistical mechanics, field theory and particle physics. His results received wide recognition and the award of the Heineman Prize in 1962, and of the Max Planck Gold medal in 1974, stand as witnesses to his achievements as a researcher.

Léon Van Hove's special talents combined excellence in theoretical physics, a deep concern for keeping a close contact between theoretical and experimental aspects and a dedication to fostering international collaboration. With considerable intelligence and tactfulness he contributed much to the fact that CERN's success is not only that of a laboratory in Geneva but of Europe as a whole.

His expertise was always in demand and beside important responsabilities at CERN he served as President of the Scientific Secretary of the Max Planck Institute in Munich and on ESO and ESA committees.

While a prominent Director of Research he remained a colleague to his friends in CERN's Theory Division and kept a strong research activity of his own until his untimely death.

Léon was a man of great honesty who expressed his opinions clearly and abhorred trivialities. Yet he was a man of dialogue, with a keen awareness of the views and needs of others. The whole world of physics has lost one of its great figures. We can but admire his full and rich life, and the example which he has set deep in all of us.

M. Jacob Geneva

\section{Letter To The Editor}

\section{Not So Obvious}

The article in your Journal based on the talk by A. Zichichi (Europhys. News 21 (1990) 116) seems to show me that theoretical thinking 60 years ago is no longer correctly understood.

How was it at the time? It was "obvious" that electrodynamics is symmetrical in $e^{ \pm}$. But this theory did not give a theory of matter, the carrier of the electric charge.

Matter consists of electrons and nuclei: the first by definition almost without mass, the second positive and massive. A convincing theory of mass did not exist - nor does it today. So it seemed that given the masses, the $e_{-}^{+}$symmetry is, as one would say today, "broken".

Taking this to be an empirical fact, the world consisted of positive protons and negative electrons. Neither quantization of the electric charge, i.e. $e^{2} / \hbar c$, nor the ratio of the masses of the proton and the electron were explained by quantum electrodynamics. Jordan, Pauli and Nietwicky had great hopes in the beginning of explaining their two numbers, 137 and 1840, using their quantum theory of electrodynamics. $e, t$ and $c$ were in a sense united by $e^{2 / \hbar c}=$ $1 / 137$ so these two numbers should spring out of the theory. As these were the hopes and speculations, protons and electrons were called "elementary particles" and it was definitely not obvious that the proton was not the "anti-electron".

This picture of the world exploded with the discovery of the neutron. Now it was clear: the rules of statistics and spin compostion are not "broken" by nuclear forces. Nor is the $e \pm$ symmetry broken by matter, all the more so with the discovery of the positron.

It seems that the notion of elementary particles is still useful: they exist in families, as in a family tree. They have many-fold symmetries, though these are mostly broken. So we have a rather strange "elementary particle model" of the world, maybe as strange as the mechanical "ether" of yonder day.

M. Fierz

Küsnacht, Switzerland 